Monatsschrift f. Geburtshülfe u. Gynäkologie 1938;108:I-IV

\title{
Contents, Vol. 108, 1938
}

\section{Inhaltsverzeichnis.}

Originalarbeiten.

Beíglböck, W., siehe unter Posatti, F.

Dorr, PL, Kasuistischer Beit Гag zur Frage der lebensretten-

den Wirku $\pi$ g des Aderlasses bei einem nach operierter

Tubarruptur aufgetretenen Lungenödem _. . 253

Fressel, D. J'., Zur Mittelscheitellage 13

Gaethgens, G., Intraabdominelle Rupturblutungen, bedingt

durch Anomalien der Plazentation 277

Gänßbauer, H., Ueber Unfruchtbarmachung durch Tubenknotung. (Mitteilung einer Verbesserung der Original-

methode.)

83

Hagedorn, W., Ueber das Durstfieber bei Neugeborenen . 247

Hellendall, H., Die stereoskopische Röntgenaufnahme zur

Lokalisation eines verlorengegangenen Intrauterinpes-

sars

143

Hollósí > K. u. Martzy, St., Ueber die Bedeutung der Blut-

bilduntersuchungen in der Gynäkologie .... 26

Hüssy, P., Einiges über Abort und Unfall 1

Jaschke, Rud. Th. von, Stillschwierigkeiten und die Wege

zu ihrer Ueberwindung

57

Kovács, F., Zum Wirkungsmechanismus der weiblichen

Sexualhormone 93

Kehrer, E., Ueber Mongolismus

- Die Bedeutung des großen Netzes in anatomischer, physiologischer und pathologischer Beziehung . . 181

Knebel, R., «Der erste Akt der manuellen Plazentarlösung» 207

Lehndorff, H., Anämische Zustände bei Neugeborenen . 264

Martzy, St., siehe Hollósí, K.

Oehlke, R., Bandwurmerkrankung als Ursache eines unstillbaren Schwangerschaftserbrechens . . . 153

Podleschka, K., Metastatische Peritonitis intra partum . 16

Posatti, F. u. Beiglböck, W., Ueber den Kalium- und Natriumgehalt des Serums bei präeklamptischen Zuständen und seine Beeinflussung durch die Insulinhypoglykämie 237

Schmidt, W., Die abdominelle Tubendurchblasung und die Implantation des Ovariums in den Uterus . . 209

- Ueber die drei Entbindungen einer Hebosteotomierten 
mit Einlagerung eines Autotransplantats . . . 211

Seebach, W., siehe unter Winkler, H.

Üstün, Z., Ueber experimentelle Transplantationen von

Ovarien

Volz, H., Erfahmngen in der Aufzucht von Frühgeburten 158

Winkler, H. u. Seebach, W., Klinische und experimentelle Beobachtungen bei Vitamin-C-

Belastungen während des Menstruationszyklus. (Zugleich ein Beitrag zur Methode der

Askorbinsäurebestimmung.) ... 67

Grenzgebiete der Geburtshilfe und Gynäkologie.

Wolf, Willi, Kreislaufbericht 1937165

Kramann, Heinrich, Die histologischen Forschungen über

die weibiichen Genitalorgane 214

Sitzungsberichte aus geburtshilflich-gynäkologischen Gesellschaften.

Nordwestdeutsche Gesellschaft für Gynäkologie. Sitzung

vom 22. Mai 1937 in Stettin 35

Gesellschaft für Geburtshilfe und Gynäkologie Berlin. Sit

zung vom 18. Juni $1937 \quad 55$

Sitzung vom 12. FebruaГ $1937 \quad 232$

Sitzung vom 16. April 1937286

Geburtshilflich-gynäkologische Gesellschaft in Wien. Sit

zung vom 8. Juni 1937

290

Buchbesprechungen Tagesnachrichten Sachregíster zu Bd. 101-108 Namenregíster zu Bd. 101108

180,236

180,236

.293

307 